\title{
Numerical simulation of two-phase partition chromatography in microchannels for moderated $\log P$ measurements
}

\author{
Sorina M. Ulmeanu ${ }^{\mathrm{a}}$, Jacques Josserand ${ }^{\mathrm{a}}$, Henrik Jensen ${ }^{\mathrm{a}}$, Géraldine Bouchard ${ }^{\mathrm{b}}$, \\ Pierre-Alain Carrupt ${ }^{\mathrm{b}}$, Hubert H. Girault ${ }^{\mathrm{a}, *}$ \\ a Laboratoire d'Electrochimie Physique et Analytique, Ecole Polytechnique Fédérale de Lausanne, CH-1015 Lausanne, Switzerland \\ ${ }^{\mathrm{b}}$ Institut de Chimie Thérapeutique, Université de Lausanne, CH-1015 Lausanne, Switzerland
}

Received 5 December 2003; received in revised form 8 April 2004; accepted 15 November 2004

Available online 10 December 2004

\begin{abstract}
A finite element simulation has been used in order to study the partition chromatography process of one species between an aqueous mobile phase and an organic stationary phase located at the bottom of a rectangular microchannel. The transient model incorporates convection-diffusion of the species in the water phase coupled to the diffusion in the stationary organic phase by the way of the partition kinetics at the interface. The time evolution of the injected species concentration is analyzed versus the velocity of the mobile phase, the detecting position and the thickness of the stationary phase. The comparison of simulation results with both experimental data and analytical model confirm its validity. These simulations show that thin channels can be used to measure $\log P$ of molecules from their retention time. Finally, we have shown how the sample velocity can be optimized for a given geometry of the channel and diffusion coefficient of the species.
\end{abstract}

(C) 2004 Elsevier B.V. All rights reserved.

Keywords: Simulation; Partition chromatography; Finite element method; Numerical model; Liquid-liquid interface; Microchannel

\section{Introduction}

Lipophilicity, expressed as $\log P$ (logarithm of the partition coefficient), is a key property in quantitative structure-activity relationship studies and in other structurebased drug-design methods. Until now, different techniques have been developed to measure the distribution of the molecules. The classical shake-flask (SF) method [1] is timeconsuming and is inappropriate outside the range -1 to $3 \log P$, and UV-detection is used for certain molecules. In recent years, new experimental techniques have become available to determine the partition coefficients of neutral and ionised forms. These include centrifugal partition chromatography $(\mathrm{CPC})[2,3]$, parallel artificial membrane permeation

\footnotetext{
* Corresponding author. Tel.: +41 21693 3145; fax: +41 216933667. E-mail address: hubert.girault@epfl.ch (H.H. Girault).
}

assay (PAMPA) [4-7], potentiometry [8,9] and amperometric techniques [10-13].

Partition chromatographic methods are inherently suited to measure the $\log P$ [14-19] as the net retention time is a direct measure of the partition coefficient for a given phase ratio. The most currently used techniques among partition chromatographic methods are liquid-liquid chromatographic techniques [20-23]. Two different classes of liquid|liquid partition chromatography can be considered. In the most common approach, the stationary liquid phase is supported on a solid-support usually by grafting long chain molecules. In the case of drug partition, $\mathrm{C}_{18}$ reversed phase HPLC $[14,24,25]$ and grafted lipid layer have been used as stationary phase in order to better mimic biological systems [26,27].

The second class of methods relies on non-supported liquid stationary phases as in centrifugal partition or as in counter droplet chromatography $[14,28,29]$. The main advantage of CPC is to yield $\log P$ values between im- 
miscible solvents such as water/alkanes, water/octanol or water/DCE.

In the present work, we propose an alternative approach where the stationary liquid phase is immobilized in a microchannel by gelification or supported by a porous phase. In the case of ions, it has been shown that the standard $\log P$ values between water and nitrobenzene remain constant when polyvinylchloride is added to the organic solvent for gelification [30,31]. We have also shown that the standard $\log P$ values between water and nitrophenyloctyl ether (NPOE) remain constant for ions when the organic phase is supported on a porous hydrophobic membrane of polyvinylidene difluoride (PVDF) [9].

The final goal of the present work is to develop a "highthroughput" $\log P$ measurement in microchips based on partition chromatography. We report here a computer simulation of liquid|liquid partition chromatography in a microchannel. The model is validated by comparing the simulated curves with both analytical and experimental ones. The present study analyzes the effects of the following parameters on the detected signal: the partition coefficient $(\log P)$, the depth of the channels, the shape and the magnitude of the fluid velocity, the detecting position and the diffusion coefficient of the organic phase. The two-phase system used for the experiments is octanol-water with 2,4-dinitrophenol as a solute in its neutral form. The results show how the adjustment of some of these parameters can be of importance to enable a good quality of the detected signal.

\section{Theory}

\subsection{Classical models}

The theory of mass transfer in chromatographic columns has been theoretically investigated since the pioneering work of Martin and Synge [32]. One of the first models considers the system as a series of ideally mixed cells [33,34]. It describes the column as a series of consecutive elementary cells in which partition processes are supposed to be at equilibrium. In this case, the partition kinetics can be written as:

$c_{1} \underset{k_{2}}{\stackrel{k_{1}}{\rightleftarrows}} c_{2}, \quad P=\frac{k_{1}}{k_{2}}=\left(\frac{c_{2}}{c_{1}}\right)_{\text {equilibrium }}$

where $c_{1,2}$ are the concentrations of the species in the water phase and organic phase, respectively, $k_{1,2}$ are the partition rate constants (or kinetic constants) at the interface and $P$ represents the solute partition coefficient between the two phases.

Continuous models [33] include the longitudinal diffusion of the solute in both phases assuming that the transversal diffusion is very fast (thin layer assumption: transversal mass transfer is fast enough compared to the other changes occurring so that the process is governed only by longitudinal dispersion of the solute in the stationary and mobile phases).
On the other hand, the equations of the kinetic model of chromatography [35] have been solved taking into account the interfacial kinetics in the absence of longitudinal diffusion in the column, for a Dirac impulse injection. This model also assumes that the transversal diffusion has reached a local steady-state (thin layer cell as above, where the species have enough time to diffuse to the lateral walls, i.e. concentrations are uniform along $y$ axis for a given length $x$ ). It assumes that:

$\frac{t_{\text {Diff }}}{t_{\text {Res }}}=\frac{\delta^{2} / D_{i}}{L_{x} / \bar{V}}=P e_{\mathrm{d}} \frac{\delta}{L_{x}} \ll 1$

where $\delta$ is the characteristic depth of the channel, $D_{i}$ is the molecular diffusion coefficient of the species in the phase $i$, $t_{\text {Diff }}$ is the characteristic transversal diffusion time, $t_{\mathrm{Res}}$ is the residence or dead time (calculated from $L_{x}$, the cell length of the microchannel), $\bar{V}$ is the mean value of the mobile phase velocity and $P e_{\mathrm{d}}$ is the transversal Péclet number as defined later in Eq. (6).

To sum up, for $t_{\text {Res }} / t_{\text {Diff }} \gg 1$ we have a uniform concentration distribution in $y$ direction (thin layer assumption), while for the other situations transient regime of diffusion has to be studied (our case). Transient kinetics have also to be taken into account because the local equilibrium at the interface is modified at each time by the diffusion.

In liquid|liquid chromatography, the distribution of a solute between the phases is determined by the partition coefficient $P$, the interphase mass transfer rates and the ratio of phase volumes. Therefore, the time evolution of the sample concentration in the channel should be modeled in order to obtain the retention time. For non-ideal chromatography, the determination of meaningful retention parameters from non-Gaussian chromatograms uses the first moment (instead of the peak of maximum concentration). This first moment method is here considered: it can produce a more accurate result because it takes into account the whole curve and not only the peak. The first moment is also called the mean value of the temporal concentration distribution $[36,37]$ and is given by the equation:

$t_{\text {Ret }}=\mu_{1}=\frac{\int_{0}^{\infty} t c(t) \mathrm{d} t}{\int_{0}^{\infty} c(t) \mathrm{d} t}$

\subsection{Numerical model}

In the model envisaged, the partition process is studied by solving the mass transport equations coupled with partition kinetics at the interface.

It leads ((4), (5)) to the transient diffusion-convection-reaction of the species partitioning at the interface:

$$
\begin{aligned}
& \frac{\partial c_{1}}{\partial t}+\nabla \cdot\left(-D_{1} \nabla c_{1}+\mathbf{V} c_{1}\right)=-k_{1} c_{1}+k_{2} c_{2} \\
& \quad \text { in mobile phase }+ \text { interface }
\end{aligned}
$$




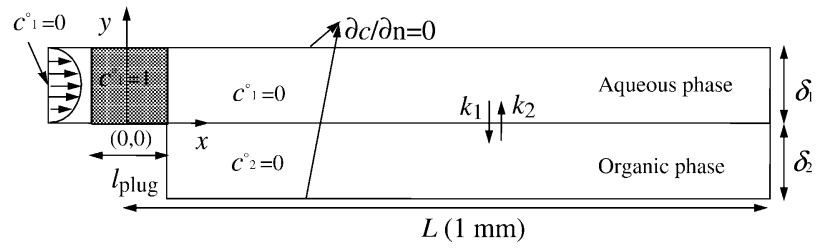

Fig. 1. The cell: geometry description, initial and boundary conditions. For all cases, excepted Fig. 9a flat profile is assumed (the parabolic flow is used to study the stretching effect of the flow profile on the detection signal). The length of the plug $l_{\text {plug }}$ is $0.1 \mathrm{~mm}$.

$$
\begin{aligned}
& \frac{\partial c_{2}}{\partial t}+\nabla \cdot\left(-D_{2} \nabla c_{2}\right)=k_{1} c_{1}-k_{2} c_{2} \\
& \text { in stationary phase }+ \text { interface }
\end{aligned}
$$

where $D_{1,2}$ are the molecular diffusion coefficients of the species in water and organic phase, respectively, $k_{1,2}$ are the partition rate constants (or kinetic constants) at the interface as defined in Eq. (1) and $\mathbf{V}$ is the velocity vector of the fluid. The transversal Péclet number in aqueous phase can be adapted to the geometry of the channel and defined as:

$P e_{\mathrm{d}}=\frac{\bar{V} \delta_{1}}{D_{1}}$

where $\delta_{1}$ is the depth of the flowing channel and $D_{1}$ is the diffusion coefficient in the water phase. The corresponding Péclet values is ranging from 1 to 20. For the following study, the transient convection-diffusion equation is solved for Reynolds numbers ranging from $10^{-3}$ to 0.02 .

The assumptions made in these simulations are as following:

(i) The first calculations are done with an uniform plug flow profile. The stretching effect [38] of the parabolic flow profile is studied at the end of the paper (see Fig. 9).

(ii) A two-dimensional model is used (see Fig. 1) to decrease the number of nodes and the calculation time. This assumes that the width $(w)$ of the channel is much larger that its depth $(w / \delta \gg 1)$ so that the velocity gradient in the third dimension can be neglected (value in experimental part is $w / \delta=50$ ).

(iii) The sample introduced in the channel contains one species.

(iv) The solution is sufficiently diluted to assume that the variations of the concentration do not modify the viscosity and the density of the fluid, which are also assumed to be uniform.

(v) The channel walls are assumed to be smooth and the interface between the water and the organic phases is assumed to be planar.

Geometries and numerical parameters. Eqs. (4) and (5) are implemented on the finite element software Flux-Expert ${ }^{\circledR}$ (Astek, France). The geometry of the cell is illustrated in Fig. 1, where the organic phase is assumed to be the sta-
Table 1

Main parameter values used in the simulations

\begin{tabular}{lll}
\hline Parameter & Values & Reference \\
\hline$\delta_{1} \delta_{1} / \delta_{2} \delta_{2}(\mu \mathrm{m})$ & $100 / 100,50 / 50$ & $50 / 50$ \\
& $20 / 20,5 / 5$ & \\
$k_{1} / k_{2}$ & $0,1,3,10\left(k_{2}=10^{3} \mathrm{~s}^{-1}\right)$ & 10 \\
$V(\mathrm{~mm} / \mathrm{s})$ & $0.01-0.075(100 / 100)$ & 0.075 \\
& $0.01-0.25(50 / 50)$ & \\
& $0.025-1(20 / 20)$ & $10^{-9}$ \\
$D_{1}\left(\mathrm{~m}^{2} \mathrm{~s}^{-1}\right)$ & $10^{-9}$ & $10^{-9}$ \\
$D_{2}\left(\mathrm{~m}^{2} \mathrm{~s}^{-1}\right)$ & $10^{-9}, 5 \times 10^{-10}, 10^{-10}, 5 \times 10^{-11}$ & \\
\hline
\end{tabular}

tionary phase and the aqueous phase is the moving one. $\delta_{1}$, $\delta_{2}$ and $L$ are defined as the channel depth (for the aqueous and organic phase) and the channel length, respectively. The origin, $(0,0)$, is fixed at the middle length of the plug (length is $=100 \mu \mathrm{m}$ ). For all the measurements, the detecting position of the species is centered at the point $\left(L, \delta_{1} / 2\right)$ at the end of the water channel $(L=1 \mathrm{~mm})$. A transversal integration path in the water channel from point $(L, 0)$ to point $\left(L, \delta_{1}\right) \mu \mathrm{m}$ has also been used. The difference between these two probes was $1 \%$ : as a consequence, the detector employed to evaluate the signal is the centered point described above.

As shown in Fig. 1, the initial conditions are: $c_{1}^{\circ}=1 \mathrm{mM}$ for the sample plug and 0 for the fresh water and organic phases. The boundary condition is $c_{1}^{\circ}=0$ at the inlet (fresh fluid arriving after the plug). The choice of the time step and mesh size was the result of a careful calibration and error evaluation as described in the supporting information (see Tables 1 and 2).

A calibration of the values of $k_{1}$ and $k_{2}$ was done in order to be independent of the kinetics rates and insure a diffusion limited regime. Therefore, the value of $k_{2}$ was varied from $10^{-6}$ to $10^{3} \mathrm{~s}^{-1}$, keeping the ratio, $k_{1} / k_{2}$, constant at 10. For $D=10^{-9} \mathrm{~m}^{2} / \mathrm{s}, \delta_{1} / \delta_{2}=100 / 100 \mu \mathrm{m}$, results are independent of the kinetics, for the $k_{2}$ value above $10^{-3} \mathrm{~s}^{-1}$. To be diffusion limited whatever the channel depth is, the set $k_{1} / k_{2}=10^{4} / 10^{3}$ is chosen as the reference case. The values of parameters used are listed in Table 1.

The time step used was $1,0.1$ and $0.01 \mathrm{~s}$ for $100 / 100$, $50 / 50$ and $20 / 20 \mu \mathrm{m}$ channels, respectively, and an optimized mesh was used for the concentration calculation (size for the smaller mesh element: 10,5 and $2 \mu \mathrm{m}$ for the cases presented above). The results show $0.12 \%$ error for the time step of $0.01 \mathrm{~s}$ compared with the time step of $0.001 \mathrm{~s}$ (see Tables 1 and 2, in supporting information).

\section{Experimental set-up}

The system consists of a "double Y" channel configuration, where the two solvent phases (water and organic) are put together to form a liquid|liquid interface. The organic phase (in this work, $n$-octanol) is first introduced in the channel by 


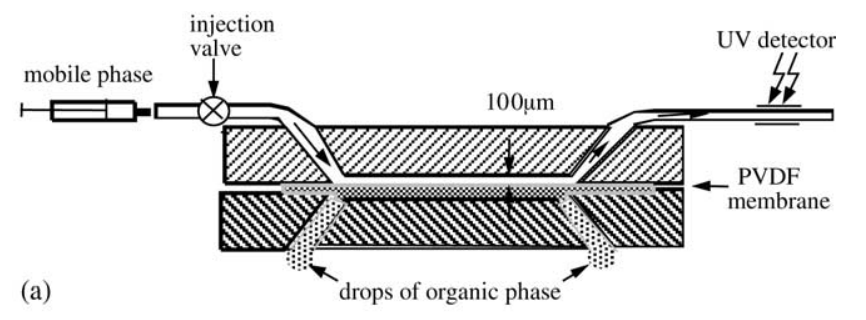

(b)

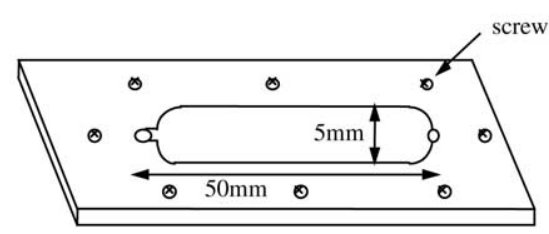

Fig. 2. Layout and dimensions of the present device with the cross section (a) and a top view of one of the two pieces (b).

using a polyvinylidene difluoride (PVDF) hydrophobic membrane. After that, the water phase is pushed in the channel with a syringe pump. The scheme is represented in Fig. 2(a) and (b). After contact with the organic phase, the aqueous mobile phase is carried away into a capillary tube and directly coupled with an UV detector. Between the cell and the syringe pump an auto-injection valve AV7-3 (BioRad, Switzerland) with a low injection volume of about $1.4 \mu \mathrm{L}$ of sample is connected.

The system was composed of two pieces, one of polycarbonate (PC) for the water phase and the second one of polyetheretherketone (PEEK) for the organic phase. These pieces were fixed together, face to face using screws. Each channel is $50 \mathrm{~mm}$ long, $5 \mathrm{~mm}$ wide, $100 \mu \mathrm{m}$ deep $(L / \delta=500$, $w / \delta=50)$.

\section{Results and discussion}

\subsection{Calibration}

The present model was compared to the analytical model given by Kuznetsov and Girault [35] for the same geometry (double microchannel with a stationary and mobile phase). In this case, the channels are very thin (depth $5 / 5 \mu \mathrm{m}$ ) to neglect the transversal diffusion. The injected sample plug is a Dirac function, in the absence of axial (i.e. longitudinal) dispersion. This Dirac function is approximated by a thin plug of thickness $l_{\text {plug }}$ equal to $0.25 \mu \mathrm{m}$ and $0.1 \mu \mathrm{m}$ (i.e. $5 \%$ and $20 \%$ of the channel depth $\delta$ ).

To illustrate better this analytical solution, let us introduce two independent dimensionless parameters - a kinetic retention coefficient $\xi$ and a dimensionless time $\tau\left(\xi=t_{\text {Res }} k_{1}\right.$, $\tau=t_{\text {Ret }} \cdot k_{2}$ ). The rate constants $k_{1,2}$ are both equal to 10 , leading to $P=1$ and the velocity $V=0.01 \mathrm{~mm} / \mathrm{s}$ (flat profile). The time evolution of the normalized (dimensionless) concentration $\left(c_{1} L P \phi / c_{1}^{\circ} l_{\text {plug }}\right)$ is represented in Fig. 3 for different values of the two independent dimensionless parameters $\xi$ and $\tau$. The concentration of injected solute $c_{1}^{\circ}$ (molar) is

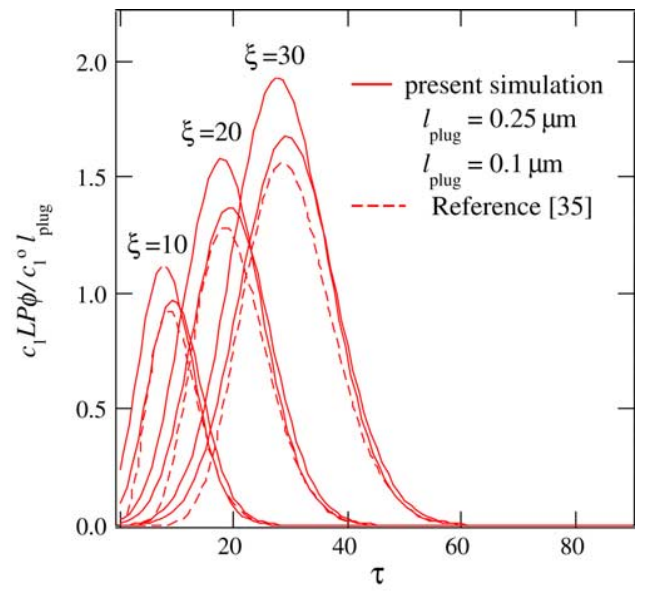

Fig. 3. Comparison of the simulated chromatographic response with analytical results [35] in the absence of axial dispersion $\left(5 / 5 \mu \mathrm{m}, k_{1} / k_{2}=1\right.$, $V=0.01 \mathrm{~mm} / \mathrm{s})$. The thickness of the plug is varied from top to bottom $(0.25$ and $0.1 \mu \mathrm{m})$.

multiplied by the length of the injection plug being as a fraction of column dead volume. The variation of $\xi$ is obtained by the variation of the detecting position $x$ along the channel. As expected, the curves obtained for the thinner plug $\left(l_{\text {plug }}=0.1 \mu \mathrm{m}\right)$ fit better with the analytical model.

\subsection{Effect of the partition coefficient $P$}

In liquid|liquid chromatography studies, the goal is to determine the partition coefficient value of a given species. Therefore, the effect of the partition coefficient on the concentration evolution at the probe is first investigated. In Fig. 4, the concentration $c_{1}$ is illustrated as a function of time for different ratios of $k_{1} / k_{2}$. For these calculations, the other parameters

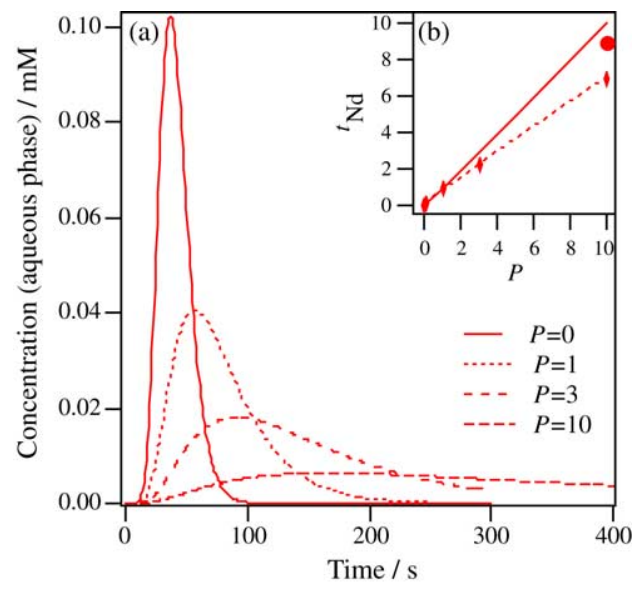

Fig. 4. (a) Effect of the partition coefficient on the time evolution of the concentration at the outlet of the channel, $x=1 \mathrm{~mm}, y=50 \mu \mathrm{m}$, $\delta_{1} / \delta_{2}=100 / 100 \mu \mathrm{m}, V=0.025 \mathrm{~mm} / \mathrm{s}$ (flat profile of the velocity). (b) Plot of the dimensionless retention time vs. the partition coefficient $(P)(-)$ theoretical line, $(\checkmark)$ numerical points for a $100 / 100 \mu \mathrm{m}$ thickness of the microchannel and ( numerical point obtained for a $20 / 20 \mu \mathrm{m}$ microchannel $(V=0.5 \mathrm{~mm} / \mathrm{s})$. 
values are kept constant (i.e. geometry: depth 100/100 $\mu \mathrm{m}$, diffusion coefficient in water and organic phase: $10^{-9} \mathrm{~m}^{2} / \mathrm{s}$, and mobile phase velocity: $0.025 \mathrm{~mm} / \mathrm{s}$ ). The $k_{1} / k_{2}$ ratio is varied from $0,1,3-10$. It is quite evident that for higher partition coefficient, the species will be retained for a longer time in the organic phase, therefore the diffusion time increases and the measured curve presents an extended tail, the curves becoming less symmetrical and very flat. As it can be observed in Fig. 4(a), the curves are delayed with increasing the $P$-value so that the retention time increases and the peak value decreases. Under the equilibrium partition assumption, the retention time is related to the partition coefficient by the following equation [32]:

$t_{\mathrm{Nd}}=\frac{t_{\text {Ret }}-t_{\text {Res }}}{t_{\text {Res }}}=P \phi$

where $t_{\mathrm{Nd}}$ represents the normalized retention time and $\phi$ is defined as the ratio between the volume of the stationary and the mobile phases, respectively, which is equal to unity in the present work. Consequently, the plot of the dimensionless retention time versus the partition coefficient gives a straight line with a slope of unity, as it can be seen in Fig. 4(b) (full line). The dotted line corresponds to the numerical results obtained with a 100/100 $\mu \mathrm{m}$ thickness of the microchannel, giving a slope value $30 \%$ below the theoretical one. That comes from the inaccurate determination of the retention time due to a very flat curve with a long tail (for $P=10$ ). The second reason is that the species have not enough time to diffuse to the wall of the organic phase and do not take advantage of the confinement parameter $\phi$. That is confirmed by the use of a thinner microchannel $(20 / 20 \mu \mathrm{m})$ for which the numerical point $\left(^{\circ}\right)$ goes up to the theoretical line $\left(t_{\mathrm{Nd}}=9\right.$ instead of 7$)$. That will be further discussed and illustrated in Fig. 6 .

The values of $k_{1} / k_{2}$ ratio chosen in this work correspond to a moderated $\log P$ values from 0 to 1 . A lot of compounds of pharmaceutical interest have the partition coefficients in this range (i.e. antipyrine, codeine, nicotine, morphine sulfate [39]). Comparison between the curves obtained for different partition constants at the interface shows clearly that solutes with high $\log P$ need an optimized geometry.

\subsection{Effect of the sample velocity for different geometries}

To enable the comparison with the experimental case, the geometry of the microchannel is here chosen to be $100 / 100 \mu \mathrm{m}$ depth. Furthermore, a shorter channel length is taken into account $(L=1 \mathrm{~mm}$ instead of $50 \mathrm{~mm}$ used in experimental part) but the ratio $l_{\text {plug }} / L$ is maintained at the same value of 10 .

The sample velocity is varied and the other parameter values are kept constant, using the values summarized in Table 1.

Fig. 5(a) illustrates the time evolution of the concentrations at the outlet of the channel for different velocity values of the aqueous phase. In the case of high velocity values $(0.075$ and $0.05 \mathrm{~mm} / \mathrm{s})$ a double peak shape can be observed, due to the fact that the initial plug is not totally transferred
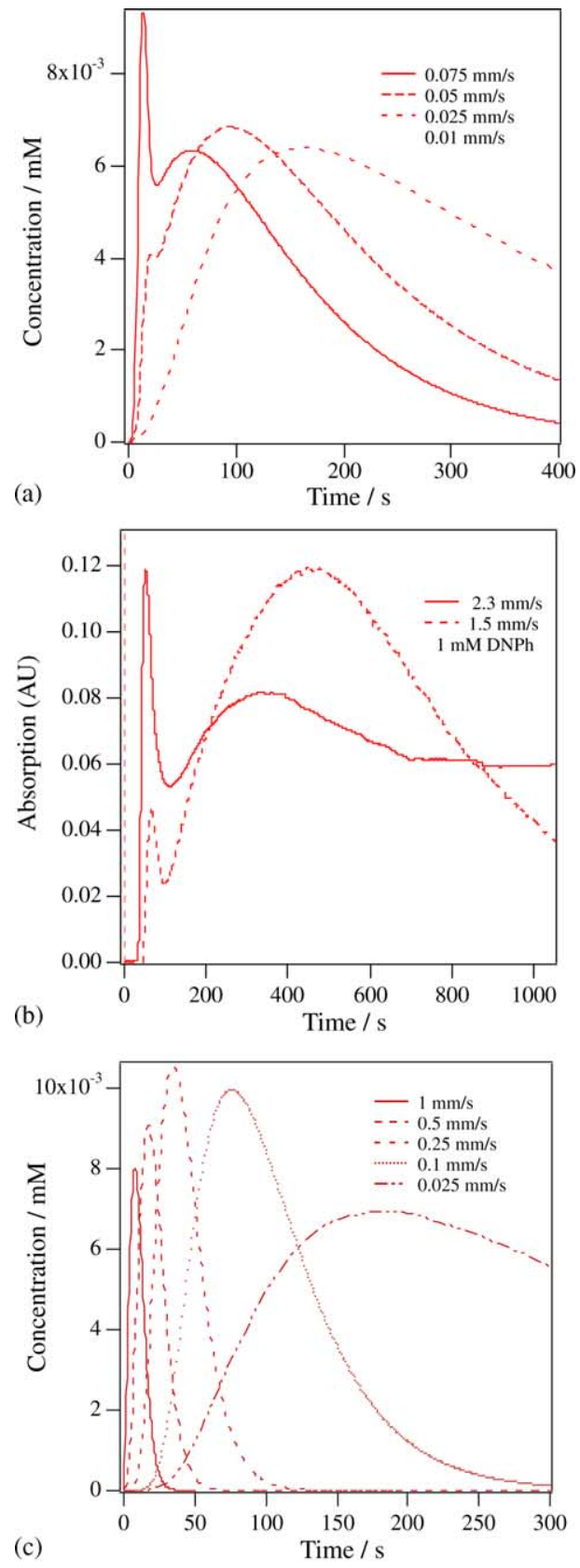

Fig. 5. (a, c) Effect of the sample velocity on the time evolution of the concentration at the outlet of the channel for the $100 / 100$ and $20 / 20 \mu \mathrm{m}$ depth channels, respectively $\left(k_{1} / k_{2}=10\right)$. (b) Experimental data obtained with the cell illustrated in Fig. 2.

into the organic phase. Indeed, the time of the first peak corresponds to the residence time, indicating that species have no time to diffuse totally to the organic phase. The second peak corresponds well to the retained species, released from the organic phase. For lower velocity value $(0.05 \mathrm{~mm} / \mathrm{s})$, we still observe the double peaks, but the initial plug almost disappears and for $0.025 \mathrm{~mm} / \mathrm{s}$, the first peak (and initial plug) disappears completely: the species have enough time to diffuse from the mobile phase to the stationary one and to return. In this case, the partition phenomena is effective and the 
response curve will be more delayed with an asymmetrical shape. When the depth of each channel is $100 \mu \mathrm{m}$ and $D$ is $10^{-9} \mathrm{~m}^{2} / \mathrm{s}$, the transversal diffusion time $\left(\delta^{2} / D\right)$ is estimated to be about $10 \mathrm{~s}$. For the velocity of $0.025 \mathrm{~mm} / \mathrm{s}$, the residence time, $t_{\text {Res }}$ is four times the value of the transversal diffusion time ( 40 s, i.e. 2 crossing of each channel for the go and return path, leading to $\left.4 t_{\text {Diff }}\right)$. Consequently, the species have enough time to cross the organic phase and to cross back to the water phase when the plug has flowed away.

At low velocity value $(0.01 \mathrm{~mm} / \mathrm{s})$, diffusion effects dominate over convection and the peak has a long tail.

The above-mentioned phenomenon has also been observed experimentally. The drug partitioning experiments are carried out using the set-up illustrated in Fig. 2 by injecting the sample (e.g. $1 \mathrm{mM}$ 2,4-dinitrophenol) in the microchannel and recording the appearance of the drug on the end of the microchannel. Fig. 5(b) shows the curves obtained at two different velocities (i.e. 1.5 and $2.3 \mathrm{~mm} / \mathrm{s}$ ). The shape of the absorption curves agrees well with that of the simulation part at high velocity. Thus, the first absorption intensity corresponds to the residence time of the species and the second peak to the species retained by the organic phase ( $n$-octanol).

The good qualitative agreement between simulation and experiments indicates that the investigations obtained using the numerical simulation can be useful to optimize the parameters for practical applications.

To study the effect of the geometry, the $20 / 20 \mu \mathrm{m}$ channel depth is envisaged. For the new geometry, Fig. 5(c) shows the time evolution of the concentration (at $x=1 \mathrm{~mm}$ ) for different magnitudes of sample velocity. The sensitivity is increased with a higher magnitude of sample velocity, and the peak is narrower. It can also be observed that all the peaks are relatively symmetrical for velocities greater than $0.1 \mathrm{~mm} / \mathrm{s}$. For a low velocity value, due to a longer diffusion time, the detected peak has a tail. For this geometry, $t_{\text {Diff }}=0.4 \mathrm{~s}$, so for $V \leq 0.5 \mathrm{~mm} / \mathrm{s}$, the residence time is five times higher than the diffusion time of the species fulfilling the previous condition, $t_{\text {Res }}>4 t_{\text {Diff. }}$ For this channel, the complementary criterion of good quality of the peak is also $t_{\text {Res }}<10 t_{\text {Diff }}$ to limit the broadening of the species.

If we compare the two geometries, it is clear that the thinner one enables narrower peaks with high sensitivity due to the fact that high velocity values can be used while respecting the time for the "forward and backward" diffusion of the species.

Fig. 6 illustrates the evolution of the normalized retention time (calculated as the first moment) versus the sample velocity for different depths of the channel. The horizontal line at unity represents the theoretical relationship corresponding to Eq. (7). For each geometry, a first increase of the relative retention time is observed when increasing the sample velocity, but for higher velocities this retention gain is dampened. The low retention time for the lowest velocities is explained by the weak difference between convection velocity and diffusion one, which is not sufficient in this case. When the species are not strongly retained by the thermodynamics

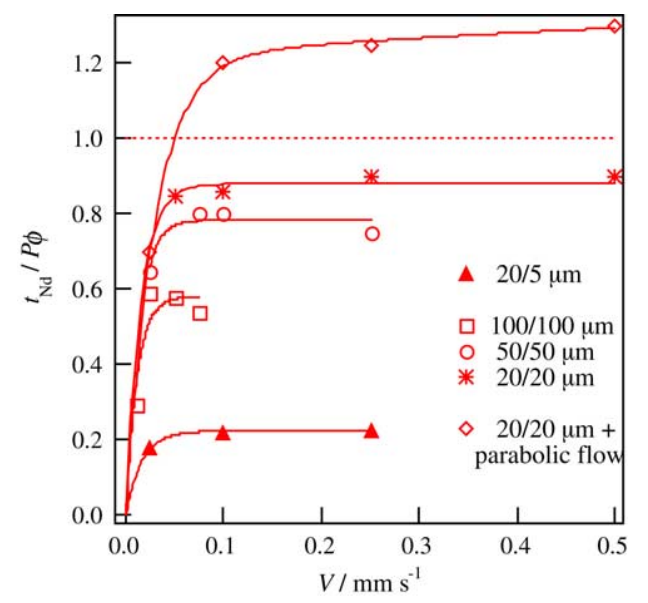

Fig. 6. Plot of non-dimensional retention times vs. sample velocity values for different depths of the microchannel. All the results were obtained for a flat flow profile except the $(\diamond)$ points that are obtained for a parabolic flow profile (see Fig. 9).

$(\log P$ around unity) and when the diffusion velocity $(D / \delta)$ is as fast as the plug flow velocity, the species have the time to go into the organic phase and come back in the mobile aqueous phase without consequent retention. Therefore, for moderated $\log P$ values, the flow velocity has to be higher than the mass transfer coefficient $D / \delta$. From this condition and the previous criterion $\left(L / V>4 t_{\text {Diff }}\right)$, it can be concluded that the velocity has to respect the following range in order to improve the retention time.

$\alpha(P) \frac{D}{\delta}<V<\frac{D L}{4 \delta^{2}}$

In other words, the sample velocity has to be chosen in such a way that species have enough time to transfer to the organic phase (for a given channel length) and in the same time the velocity has to be sufficiently high to be sure that the initial plug really overtakes the retained species (for a $\log P$ around unity). On the other hand, for high $\log P$ values, even if the species have the time to diffuse back into the mobile phase, they should wait that the concentration in the aqueous phase decrease sufficiently to transfer according to the partition coefficient (e.g. $P=1000$ ). But this extra time induce a consequent damage of the peak quality due to the diffusion and, as a consequence, high $\log P$ simulations would require much thinner geometries. The $\alpha$ parameter is introduced here to define the above relationship also for high $\log P$. With $\alpha=1$ and $\log P=1,95 \%$ of the optimal retention time is ensured for the $20 / 20$ depth of the geometry. For $\log P=2$, simulations have shown that $73 \%$ of the optimal retention time is achieved: which requires a higher value of $\alpha$ to insure $95 \%$ of the optimum retention time.

Applied to the $20 / 20 \mu \mathrm{m}$ channel, this criterion gives the velocity range $(0.1-0.5 \mathrm{~mm} / \mathrm{s})$, which is globally confirmed by Fig. 6. For the situation of near-Gaussian responses, it has also been verified that the optimum velocity given by the Van Deemter curve (based on the theoretical plate height 
representation versus the flow velocity) is comprised in the velocity range given by the criterion.

This retention decrease is particularly marked for the deeper channel $(100 / 100 \mu \mathrm{m})$ for which the optimal velocity range is narrow $(0.025 \mathrm{~mm} / \mathrm{s})$. On the other hand, the thinner channel offers a quasi plateau shape and wide range of velocity values. Moreover, for the thinner geometry $(20 / 20 \mu \mathrm{m})$, the net value of the retention time reaches eight times $t_{\text {Res }}$ and all these velocity values $(0.1<V<0.5 \mathrm{~mm} / \mathrm{s})$ have a good quality and a good retention. For the same geometry but in the case of parabolic flow profile, the retention time results $((\diamond)$ points in Fig. 6) are higher than the theoretical value due to the increase of the peak delay as it will be explained in Fig. 9.

So, the thinness of the channel improves both the peak quality and the relative retention time. On the other hand, the channel with a thinner organic phase (20/5) loses the gain of retention time (Fig. 6). Due to the phase ratio and the resulting higher local accumulation, the species in organic phase can very rapidly overcome the $P$-value (i.e. $c_{2} / c_{1}>10$ for the present case) and transfer back to the flow channel without consequent retention. However, this channel can be useful for the studies of drug with a high $P$. It is worth noticing that for each channel thickness, the maximum velocity values are limited by the time needed to diffuse to the walls. A global conclusion is that the depth of the channels is a very important parameter for the response of the detecting signal.

\subsection{Effect of the detecting position}

The processes of partition can be followed directly along the microchannel as the detection point is moved along the $x$-axis. This effect is studied for two geometries, 50/50 and $20 / 20 \mu \mathrm{m}$ via the time response of the concentration (at various $x$ positions) in the mobile phase. Fig. 7(a) shows the signal peaks at $x=0.25,0.5,0.75$ and $1 \mathrm{~mm}$ for a geometry of 50/50 $\mu \mathrm{m}$ depth. At the beginning of the microchannel, a high peak signal was observed, but the peak is rapidly damped with the distance, the shape presenting a long tail at the outlet. In Fig. 7(b), the same calculation has been done for a $20 / 20 \mu \mathrm{m}$ depth microchannel. In this case, the distribution curves are approximately symmetrical and almost without tail.

\subsection{Effect of the diffusion coefficient}

In $\log P$ experiments, the diffusion coefficient of the organic phase is strongly dependent on the material. Fig. 8 illustrates the time evolution of the concentration for a fixed velocity $(0.075 \mathrm{~mm} / \mathrm{s})$ and geometry $(50 / 50 \mu \mathrm{m})$ for different diffusion coefficient values in the organic phase. It is observed that the diffusion coefficient has not an important effect on the retention time and on the shape of the response curves. Two antagonist phenomena can be observed: the decrease of the diffusion coefficient involves a small increase of the diffusion time (i.e. a delay of the peak) but when $D_{2}$ is very
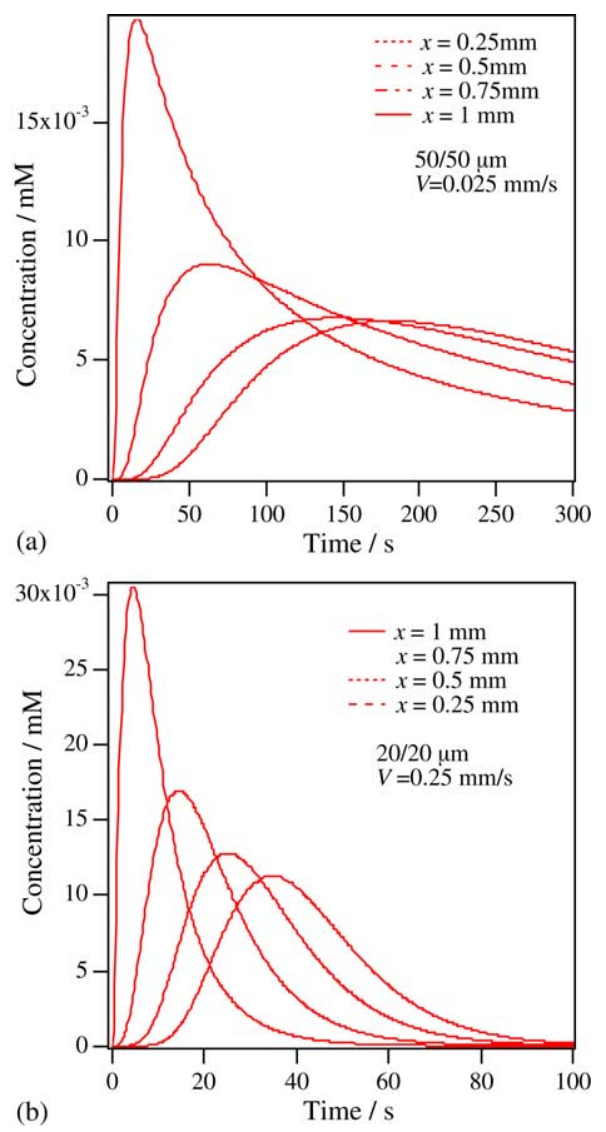

Fig. 7. Effect of the detecting position for two geometries depths (a) $50 / 50 \mu \mathrm{m}(V=0.025 \mathrm{~mm} / \mathrm{s})$ and (b) $20 / 20 \mu \mathrm{m}(V=0.25 \mathrm{~mm} / \mathrm{s})$.

low, the depth of penetration of the species in the organic phase is notably reduced, leading to a more rapid response. The first effect dominates for $D_{2}$ values between $10^{-9}$ and $10^{-10} \mathrm{~m}^{2} / \mathrm{s}$ (short delay) and the second one takes the advantage with $D_{2}=5 \times 10^{-11} \mathrm{~m}^{2} / \mathrm{s}$, for which the peak presents a little hump in its first part of the curve.

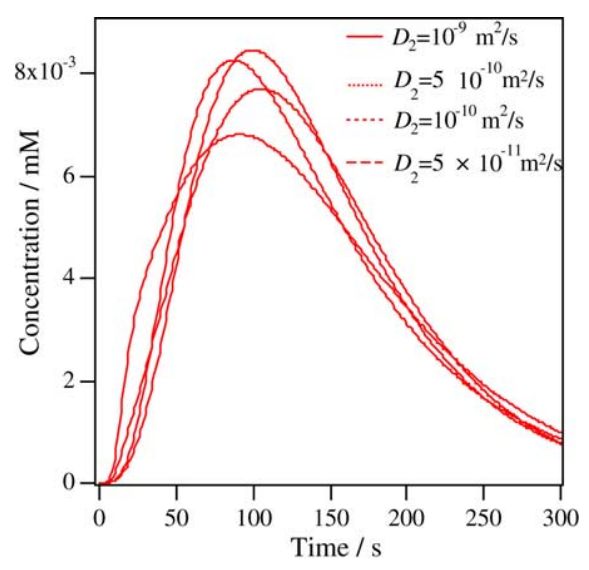

Fig. 8. Effect of the diffusion coefficient of the species in the stationary organic phase $\left(D_{2}\right)$ on the time evolution of the sample concentration, for the geometry depth of $50 / 50 \mu \mathrm{m}$. The sample velocity is $0.075 \mathrm{~mm} / \mathrm{s}$. 


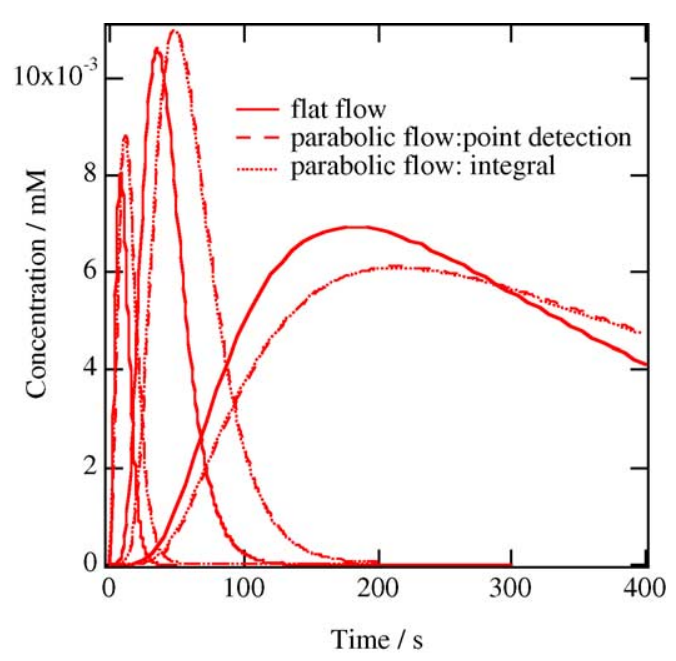

Fig. 9. Time evolution of the concentration for different sample velocities $(0.025,0.25,1 \mathrm{~mm} / \mathrm{s})$ and different integral concentration probes (the channel depth is $20 / 20 \mu \mathrm{m}$ ). Line curves correspond to the flat flow velocity. Dotted curves correspond to parabolic flow velocity for a single point detection and a transversal path detection from $\left(10^{3}, 0\right) \mu \mathrm{m}$ to $\left(10^{3}, 20\right) \mu \mathrm{m}$.

A practical consequence is that different solvents can be used as organic phase without strong influence on the final results. It is important to note that even gels could be envisaged as a stationary phase in this system.

\subsection{Effect of the flow profile}

Fig. 9 shows the time evolution of the concentration for different flow profiles, uniform and parabolic ones, respectively. For the parabolic flows, the concentration is measured in one point and is also integrated on a transversal path. It can be observed that the detection does not affect the results, the point and transversal integration giving the same results. The double humped peak effect observed in flow injection analysis (FIA) with pressure driven flow [38] does not appear in the present model. Indeed, the transfer to the static organic phase strongly damps the drawbacks of the parabolic flow. The comparison between both flows shows how the parabolic one delays and slightly broadens the detection peak. The species that come back from the organic phase arrive in the low velocity layers of the parabolic flow: as a consequence, they arrive later to the detector.

\section{Conclusion}

Simulation of liquid|liquid partition chromatography in a microchannel has been performed using the finite element method. The comparison with experimental data and analytical results showed the validity of the present model.

It is found that the partition coefficient of the species and the geometry of the microchannel contribute to major changes of the final detection signal. Indeed, a small depth of the microchannel permits a higher sample velocity and contributes to the quality of the peaks. To ensure a sufficient relative retention time, the sample velocity has to be adjusted following the criterion, $\alpha(P) D / \delta<V<L D / 4 \delta^{2}$. This criterion is valid for a moderated $\log P$ values and therefore, the sample velocity can be calculated for a given channel length or inversely. The sample velocity has to be chosen in such a way that species have enough time to transfer to the organic phase (for a given channel length). In the same time the velocity has to be sufficiently high to be sure that the initial plug really overtakes the retained species. For a 20/20 $\mu \mathrm{m}$ depth channel, the retention time is nine times higher than the residence one, for a very large range of velocity values (compared to 6 for a small velocity range for the $100 / 100 \mu \mathrm{m}$ depth microchannel). For the situation of near-Gaussian responses, it has also been verified that the velocity given by the criterion is in good agreement with the optimum velocity given by the Van Deemter approach.

When an asymmetrical microchannel is used $\left(\delta_{1} / \delta_{2}=0.2\right)$ for moderated values of $P$, the efficiency of the retention considerably decreases. It has also been demonstrated that the disturbing effects of the parabolic flow (generally observed in FIA) are strongly reduced by the exchange with the static organic phase. In addition, the low effect of $D$-values in the organic phase on the signal (for $D_{2}<10^{-9} \mathrm{~m}^{2} / \mathrm{s}$ ) encourages the use of different solvents as stationary phase. Following this, it would be interesting to study the partition of the species at water|gel interfaces.

\section{Acknowledgements}

H.G. and P.-A.C. are grateful for financial support by the Swiss National Science Foundation (Grant No. 20067727.02/1).

\section{Appendix A}

The local expressions of the flux conservation (Eqs. (4) and (5)) are derived in the global form ((A.3) and (A.4)) by using the Galerkin's formulation (multiplication by a projective function $\alpha$ and integration on the domain of study, $\Omega$ ):

$\iint_{\Omega} \alpha\left[\frac{\partial c_{i}}{\partial t}+\nabla \cdot\left(-D_{i} \nabla c_{i}+V c_{i}\right) \pm k_{i} c_{i}\right] \mathrm{d} \Omega=0$

The convection term of (A.1) is derived by taking into account the continuity equation $\nabla \cdot V=0$. By decomposing the product between $\alpha$ and the divergence, the second order derivative of (A.1) (divergence of the gradient) can be written as:

$\alpha \nabla \cdot\left(-D_{i} \nabla c_{i}\right)=\nabla \cdot\left(-\alpha D_{i} \nabla c_{i}\right)+D_{i} \nabla \alpha \cdot \nabla c_{i}$

Applying (A.2) in (A.1) and using the Ostrogradsky theorem, the divergence term is rejected at the boundary of (A.3) and 
(A.4), where it expresses the flux boundary condition of each species. This boundary condition is here equal to zero (no flux at the boundaries of the domain, excepted the inlet and outlet of the channel which are submitted to the convection fluxes).

$$
\begin{aligned}
& \iint_{\Omega}\left[\alpha \frac{\partial c_{1}}{\partial t}+D_{1} \nabla \alpha \cdot \nabla c_{1}+\alpha \mathbf{V} \cdot \nabla c_{1}+\alpha k_{1} c_{1}-\alpha k_{2} c_{2}\right] \\
& \times \mathrm{d} \Omega=0 \\
& \iint_{\Omega}\left[\alpha \frac{\partial c_{2}}{\partial t}+D_{2} \nabla \alpha \cdot \nabla c_{2}+\alpha \mathbf{V} \cdot \nabla c_{2}-\alpha k_{1} c_{1}+\alpha k_{2} c_{2}\right] \\
& \quad \times \mathrm{d} \Omega=0
\end{aligned}
$$

\section{Appendix B. Supplementary data}

Supplementary data associated with this article can be found, in the online version, at doi:10.1016/j.chroma. 2004.11.050.

\section{References}

[1] A. Hersey, A.P. Hill, R.M. Hyde, D.J. Livingstone, Quant. Struct.Act. Relat. 8 (1989) 288.

[2] G. Caron, P. Gaillard, P.A. Carrupt, B. Testa, Helv. Chim. Acta 80 (1997) 449.

[3] G. Caron, G. Steyaert, A. Pagliara, F. Reymond, P. Crivori, P. Gaillard, P.A. Carrupt, A. Avdeef, J. Comer, K.J. Box, H.H. Girault, B. Testa, Helv. Chim. Acta 82 (1999) 1211.

[4] M. Kansy, F. Senner, K. Gubernator, J. Med. Chem. 41 (1998) 1007.

[5] F. Wohnsland, B. Faller, J. Med. Chem. 44 (2001) 923.

[6] C.Y. Zhu, L. Jiang, T.M. Chen, K.K. Hwang, Eur. J. Med. Chem. 37 (2002) 399.

[7] L. Di, E.H. Kerns, K. Fan, O.J. McConnell, G.T. Carter, Eur. J. Med. Chem. 38 (2003) 223.

[8] G. Bouchard, P.A. Carrupt, B. Testa, V. Gobry, H.H. Girault, Chem.Eur. J. 8 (2002) 3478.

[9] S.M. Ulmeanu, H. Jensen, G. Bouchard, P.A. Carrupt, H.H. Girault, Pharm. Res. (2003).

[10] G. Bouchard, A. Pagliara, P.A. Carrupt, B. Testa, V. Gobry, H.H. Girault, Pharm. Res. 19 (2002) 1150.

[11] F. Reymond, G. Steyaert, P.A. Carrupt, B. Testa, H.H. Girault, J. Am. Chem. Soc. 118 (1996) 11951.
[12] F.A. Reymond, Thesis in Departament of Chemistry at Ecole Polytechnique Fédérale de Lausanne, Ecole Polytechnique Fédérale de Lausanne, Lausanne, 1997, p. 385.

[13] V. Gobry, S. Ulmeanu, F. Reymond, G. Bouchard, P.A. Carrupt, B. Testa, H.H. Girault, J. Am. Chem. Soc. 123 (2001) 10684.

[14] B. Testa, P.A. Carrupt, P. Gaillard, R.S. Tsai, in: V. Pliska, B. Testa, H. van de Waterbeemd (Eds.), Lipophilicity in Drug Action and Toxicology, VCH Publishers, Weinheim, 1996, p. 49.

[15] B. Testa, G. Caron, P. Crivori, S. Rey, M. Reist, P.A. Carrupt, Chimia 54 (2000) 672.

[16] A. Berthod, A.I. Mallet, M. Bully, Anal. Chem. 68 (1996) 431.

[17] M.C. Garcia-Alvarez-Coque, J.R. Torres-Lapasio, J.J. Baeza-Baeza, Anal. Chim. Acta 324 (1996) 163.

[18] K. Kaczmarski, W. Prus, T. Kowalska, JPC-J. Planar Chromatogr.Mod. TLC 12 (1999) 175.

[19] K. Kaczmarski, W. Prus, T. Kowalska, J. Chromatogr. A 869 (2000) 57.

[20] W. Prus, T. Kowalska, JPC-J. Planar Chromatogr.-Mod. TLC 8 (1995) 288.

[21] M.J. van Buel, L.A.M. van der Wielen, K. Luyben, J. Chromatogr. A 773 (1997) 13.

[22] M.J. van Buel, F.E.D. van Halsema, L.A.M. van der Wielen, K. Luyben, AICHE J. 44 (1998) 1356.

[23] J.L. den Hollander, B.I. Stribos, M.J. van Buel, K. Luyben, L.A.M. van der Wielen, J. Chromatogr. B 711 (1998) 223.

[24] C. Stella, P. Seuret, S. Rudaz, P.A. Carrupt, J.Y. Gauvrit, P. Lanteri, J.L. Veuthey, Chimia 57 (2003) 210.

[25] C. Stella, P. Seuret, S. Rudaz, P.A. Carrupt, J.Y. Gauvrit, P. Lanteri, J.L. Veuthey, J. Sep. Sci. 25 (2002) 1351.

[26] A. Taillardat-Bertschinger, F. Barbato, M.T. Quercia, P.A. Carrupt, M. Reist, M.I. La Rotonda, B. Testa, Helv. Chim. Acta 85 (2002) 519.

[27] A. Taillardat-Bertschinger, A. Galland, P.A. Carrupt, B. Testa, J. Chromatogr. A 953 (2002) 39.

[28] R.S. Tsai, P.A. Carrupt, B. Testa, Modern Countercurrent Chromatogr. 593 (1995) 143.

[29] N. Eltayar, R.S. Tsai, P. Vallat, C. Altomare, B. Testa, J. Chromatogr. 556 (1991) 181.

[30] H.J. Lee, P.D. Beattie, B.J. Seddon, M.D. Osborne, H.H. Girault, J. Electroanal. Chem. 440 (1997) 73.

[31] F. Reymond, H.J. Lee, J.S. Rossier, L. Tomaszewski, R. Ferrigno, C.M. Pereira, H.H. Girault, Chimia 53 (1999) 103.

[32] A.J.P. Martin, R.L.M. Synge, Chromatogr. Amino-Acids (1941).

[33] A.E. Kostanian, J. Chromatogr. A 973 (2002) 39.

[34] J. Villermaux, J. Chromatogr. Sci. 12 (1974) 822.

[35] A.M. Kuznetsov, H.H. Girault, Helv. Chim. Acta 80 (1997) 1176.

[36] E. Grushka, M.N. Myers, J.C. Giddings, Anal. Chem. 42 (1970) 21.

[37] V.B. Di Marco, G.G. Bombi, J. Chromatogr. A 931 (2001) 1.

[38] X.X. Bai, J. Josserand, H. Jensen, J.S. Rossier, H.H. Girault, Anal. Chem. 74 (2002) 6205.

[39] F. Lombardo, M.Y. Shalaeva, K.A. Tupper, F. Gao, J. Med. Chem. 44 (2001) 2490. 